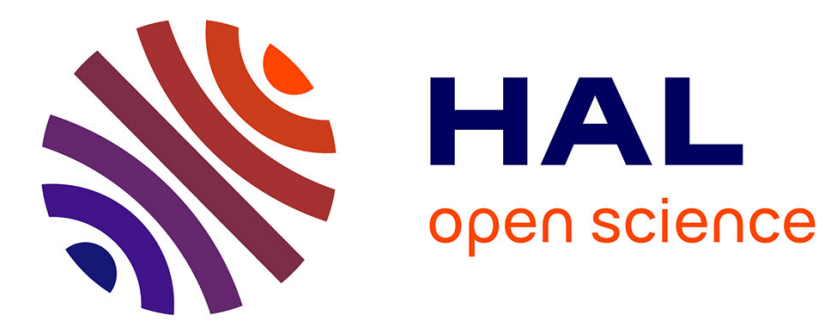

\title{
La préposition "jusqu'à", marqueur aspectuel de télicité
} Andrée Borillo

\section{To cite this version:}

Andrée Borillo. La préposition "jusqu'à", marqueur aspectuel de télicité. Faits de langues, 2014. hal-02104440

\section{HAL Id: hal-02104440 \\ https://hal.science/hal-02104440}

Submitted on 19 Apr 2019

HAL is a multi-disciplinary open access archive for the deposit and dissemination of scientific research documents, whether they are published or not. The documents may come from teaching and research institutions in France or abroad, or from public or private research centers.
L'archive ouverte pluridisciplinaire HAL, est destinée au dépôt et à la diffusion de documents scientifiques de niveau recherche, publiés ou non, émanant des établissements d'enseignement et de recherche français ou étrangers, des laboratoires publics ou privés. 


\title{
La préposition jusqu'à, marqueur aspectuel de dy- namicité
}

\author{
Andrée Borillo*
}

\section{INTRODUCTION}

L'étude proposée ici examine les propriétés attachées au SP [jusqu'à SN] lorsqu'au sein du prédicat de la phrase, celui-ci constitue un complément ayant pour objet de fixer comme terme, soit un point d'aboutissement dans le cours d'un déroulement temporel, soit un degré considéré comme très élevé (sinon le degré le plus élevé) dans une échelle de gradation quantitative :

(1) Il dit vouloir poursuivre sa grève de la faim jusqu'à sa libération

(2) C'est un homme extrême en toutes choses, il aime et il hait jusqu'à la fureur

Cette étude ignore d'emblée d'autres emplois de jusqu'à, que nous nous contentons simplement de rappeler :

a) La préposition jusqu'à, en construction avec un SN de sens spatial, peut marquer le point d'aboutissement d'une trajectoire spatiale ou d'un déplacement spatial, que ce déplacement soit réel ou fictif (cf. Borillo 2010, Fortis 2006) :

(3) Il faut suivre la route et grimper jusqu'au village, point de départ de votre randonnée.

(4) La vallée est étroite, mais des sentiers La vallée est étroite, mais des sentiers escaladent les pentes de tous les côtés de tous les côtés

b) La préposition jusqu'à, marque une borne sur un parcours temporel ou spatial. En couplage avec depuis ${ }^{l}$, elle découpe un intervalle dont elle fixe la borne droite $^{2}$ (point d'aboutissement). Dans un emploi temporel, elle peut opérer avec un $\mathrm{SN}$ de localisation figurant une borne très précise ou au contraire une délimitation assez vague, ex. jusqu'à 8h ce matin, jusqu'au soir, jusqu'à nos jours, etc. :

\footnotetext{
* CLEE/ERSS, Université Toulouse-Le Mirail, email : aborillo@univ-tlse2.fr

${ }^{1}$ Depuis est substituable par de, à partir de, comme jusqu'à peut l'être par à.

${ }^{2}$ Ce couple depuis (...) jusqu'à (...) se retrouve aussi bien pour le découpage d'un intervalle temporel que d'un intervalle spatial ex. depuis l'Atlantique jusqu'à l'Oural, depuis les pieds jusqu'à la tête, etc.
} 
(5) Découvrez à travers cette émission les talents qui ont illustré le jazz depuis ses débuts jusqu'à nos jours.

Nous mentionnons ici ce type de découpage temporel car pour figurer les deux bornes d'un intervalle, il peut être fait appel, non pas à des Noms de localisation temporelle, mais à des Noms prédicatifs, généralement dérivés de verbes, qui appartiennent précisément à la catégorie de noms que nous voulons examiner ici dans le cadre de cette étude (cf. $\$ 1$ et $\$ 2$ infra). Nous indiquons tout de suite que cette construction [depuis $\mathrm{SN}_{1}$ jusqu'à $\mathrm{SN}_{2}$ ], même quand elle fait intervenir des Noms prédicatifs qui peuvent indirectement fournir une localisation temporelle ${ }^{3}$ et signifier « le moment de $\mathrm{SN}$ » (6), n'entre pas dans l'étude que nous entendons faire ${ }^{4}$ ici :

(6) Il convient d'assurer une continuité depuis la définition d'un processus jusqu'à son application effective

c) L'adverbe jusqu'à, dans un emploi d'intensifieur argumental, peut s'appliquer à un nom dont le référent, placé dans une série construite selon un ordre décroissant de probabilité, est considéré comme marquant le point extrême de cette gradation. "Jusque projette le constituant régi au plus haut degré d'une échelle ou ordre" d'invraisemblance croissante" (cf. Fortis 2006 : 143). On retrouve là, avec jusqu'à, les propriétés d'intensification qui, dans des constructions assez similaires, ont pu être attribuées à même (voir notamment, Anscombre 1973, Ducrot 1980) :

(7) Le président fédéral change chaque année, si bien que beaucoup de citoyens suisses ignorent jusqu'à son nom.

Pour en venir à notre propos, nous voulons nous focaliser ici sur l'aspect de dynamicité que peut avoir la préposition jusqu'à, lorsque le SN régi est constitué par un nom prédicatif ou un nom abstrait. Pour examiner cet emploi, nous avons choisi d'examiner deux types de structure de phrase comportant chacune un complément de la forme [jusqu'à $\mathrm{SN}$ ] : la première fait l'objet de l'étude du $\S 1$ «jusqu'à et l'aboutissement d'un déroulement temporel »- et la seconde celle du $\S 2$ - «jusqu'à et l'accession au plus haut degré d'une échelle quantitative ».

Nous procéderons à deux études séparées dans la mesure où les deux structures de phrase étudiées respectivement au $\S 1$ et au $\S 2$, ont des propriétés syntaxiques et sémantiques suffisamment distinctes pour qu'un traitement spécifique soit réservé à chacune. Mais en même temps, ces deux structures révèlent certains points communs qui les rapprochent et qui tendent même parfois à les faire con-

\footnotetext{
${ }^{3}$ Ces noms renvoient à des événements et de ce fait acceptent de se construire avec des prépositions comme à , lors de, dès, depuis, jusqu'à, etc..

${ }^{4}$ Ceci ne veut pas dire qu'il n'y aurait pas une comparaison à faire entre les Noms prédicatifs que l'on rencontre dans cette construction avec jusqu'à et les Noms prédicatifs dont les propriétés seront définies plus loin au $\S 1$ et $\S 2$.
} 
verger. Si bien qu'après avoir décrit séparément les deux schémas de construction et expliqué l'interprétation différente que suggère l'emploi de jusqu'à dans l'un et dans l'autre cas, nous examinerons certains couples de phrases dont l'interprétation paraît relativement proche. Par exemple, nous pouvons le voir sur les deux phrases présentées ci-dessous, qui contiennent le même SP [jusqu'à l'évanouissement] :

(8) Ils se mirent tous ensemble à la rouer de coups jusqu'à son évanouissement.

(9) J'avais de nausées, des vertiges ou je faisais carrément des malaises jusqu'à l'évanouissement (...).

\section{JUSQU'A ET L'ABOUTISSEMENT D'UN DEROULEMENT TEMPOREL}

Nous choisissons de nous focaliser ici sur la construction [jusqu'à $\mathrm{SN}$ ] où la tête du SN régi est un Nom prédicatif qui marque le terme d'un déroulement temporel. Nous écartons donc l'emploi de jusqu'à avec un SN ou un Adverbe de localisation temporelle $\mathrm{e}^{5}$ (ex. maintenant, alors, là, ici, plus tard, etc ), construction qui avec certains verbes qui expriment un décalage dans le temps (remettre $\grave{a}$, remonter $\grave{a}$, reporter $\grave{a}$, etc.) peut remplir la fonction de complément argumental :

(10) Il faut repousser la réunion jusqu'à demain

(11) Il était capable de remonter jusqu'à plus de deux cents ans

Mais, dans la majorité des cas, le SP s'emploie dans la fonction de complément circonstanciel (ou complément disjoint) :

(12) Je suis ici pour longtemps, peut-être jusqu'à la fin de la guerre

(13) Arrivait un moment où ils faiblissaient. Il fallait tenir jusque là

Par ailleurs, avant d'entamer cette étude centrée sur le Nom prédicatif (Npred) qui, dans [jusqu'à SN], représente le point d'aboutissement d'un déroulement temporel, nous devons indiquer que ce point d'aboutissement peut également être représenté par une proposition infinitive [Jusqu'à Infinitive] ou par une phrase [jusqu'à ce que $\mathrm{P}]$ :

(14) Mélanger puis pétrir la pâte jusqu'à obtenir une boule homogène

(15) La décision des autorités est de détenir indéfiniment les migrants jusqu'à leur retour forcé ou jusqu'à ce qu'ils consentent à un retour volontaire

\footnotetext{
${ }^{5}$ La localisation temporelle de la borne droite (point d'aboutissement) peut être indirectement retrouvée par un calcul de durée effectué à partir du moment d'énonciation, par ex. jusqu'à hier, jusqu'il y a trois jours, etc. (parfois de manière très approximative, ex., jusqu'à il y a peu,) :.
} 
Nous n'entendons pas traiter ici de ces deux types de structure ${ }^{6}$. Nous nous contenterons d'introduire brièvement [jusqu'à Infinitive] plus loin au $\$ 2$ pour montrer que la construction infinitive se révèle d'un emploi très courant quand il s'agit d'exprimer l'accession au sommet d'une échelle quantitative. Pour ce qui est de l'emploi de [jusqu'à Infinitive] pour marquer le point d'aboutissement d'un déroulement temporel, nous la considérerons simplement comme une variante de [jusqu'à $\mathrm{SN}$ ] si le nom régi peut être considéré comme dérivé du verbe en question ; ce qui est le cas de la phrase (14) ci-dessus, à rapprocher de la version nominale représentée par (14') :

(14') Mélanger puis pétrir la pâte jusqu'à l'obtention d'une boule homogène

Si nous nous basons sur un ensemble assez large de phrases que nous avons recueillies 8 , l'une des caractéristiques de ce type de complément [jusqu'à Infinitive] est d'être assez communément utilisé dans des descriptions d'expérience ou dans des discours prescriptifs (modes d'emploi). Ceci, peut-être, afin de donner un caractère plus concret à la présentation des opérations qui se succèdent et constituent les différentes phases d'une procédure d'ensemble ${ }^{9}$ :

(16) Tirez l'anneau orange (situé sur le côté gauche de chaque cartouche) vers le haut jusqu'à retirer complètement l'adhésif hermétique.

Dans cette étude, nous avons donc fait le choix, pour l'étude du SP[jusqu'à $\mathrm{SN}$ ] utilisé dans la fonction de complément circonstanciel, de nous intéresser aux cas où la tête du $\mathrm{SN}$ régi est un nom prédicatif (Npred), comme dans l'exemple (1) déjà mentionné ou (17) ci-dessous :

(1) Il dit vouloir poursuivre sa grève de la faim jusqu'à sa libération

(17) Lascaux fut ouverte au public pendant de nombreuses années jusqu'à sa fermeture en 1963 .

\subsection{Les conditions d'ordre aspectuel liées à l'emploi du SP [jusqu'à SN]} comme complément circonstanciel

Pour que le SP[jusqu'à SN] puisse être interprété comme un point d'aboutissement dans un déroulement temporel, il faut que certaines propriétés soient présentes dans les deux structures mises en relation dans la phrase, a) les unes concernant le prédicat verbal ou nominal auquel le SP [jusqu'à SN] se rat-

\footnotetext{
${ }^{6}$ Il est certain qu'une description serait à faire de ces deux types de construction pour donner une plus grande ampleur à l'étude que nous présentons ici.

${ }^{7}$ Dans l'exemple (15), il n'y a pas de nom dérivé correspondant au verbe (retrouver).

${ }^{8}$ Les exemples qui sont proposés dans cette étude sont tous des énoncés attestés, relevés dans la base Frantext (ATILF Nancy) ou sur Internet (grâce au moteur de recherche Google).

${ }^{9}$ Il serait bon d'examiner ceci plus précisément sur des corpus de textes représentatifs de ce type de discours.
} 
tache comme complément, b) les autres concernant le $\mathrm{N}$, tête du SN régi par jusqu'à.

a) Pour le prédicat verbal ou nominal dont dépend syntaxiquement [jusqu'à $\mathrm{SN}$ ], la condition est assez simple : il doit pouvoir être affecté du trait [+duratif], que ce trait aspectuel soit inhérent au verbe (ou au nom prédicatif) qui en est la tête, ou qu'il soit le résultat de facteurs dû aux éléments participant à sa construction (temps du verbe, auxiliaires verbaux, adverbes, etc.) ${ }^{10}$.

Parmi les verbes ou les noms prédicatifs ayant le trait [+ duratif], on peut compter non seulement tous les verbes et les noms de type statif (V: être ${ }^{11}$, rester, (se) maintenir, durer, etc.; $\mathrm{N}$ : maintien, conservation, garde, etc.) mais aussi, de très nombreux verbes et noms de type dynamique - la durée pouvant être traitée en continu ex. (18) - (19) ou en discontinu (itération, fréquence) ex. (20) - (21) :

(18) Cette action doit démarrer avant le lancement du programme et se maintenir jusqu'à sa clôture

(19) Le conditionnement, c'est ce qui permet de contenir le produit, de le stocker et d'assurer sa conservation jusqu'à sa consommation

(20) Je voyais régulièrement mon cousin jusqu'à sa disparition mais je me rends compte que je ne le connaissais pas aussi bien que vous

(21) Nous réduisons votre franchise tous les ans jusqu'à sa disparition complète au bout de 5 ans

b) Pour le SN régi par jusqu'à, le nom-tête doit nécessairement être doté du trait aspectuel [+dynamique], trait qui définit, au premier niveau, tous les noms dénotant des événements, des actions, des procès, que nous regroupons sous le terme Noms d'Eventualité ${ }^{12}(\mathrm{Nev})$.

Pour mieux caractériser les noms à l'intérieur de la catégorie des Nev, il convient de faire intervenir d'autres traits : en effet, comme pour les verbes, les propriétés d'aspect que l'on peut attribuer aux noms prédicatifs, sont liées d'une part à la notion de "dynamicité", d'autre part, à la condition de "délimitation temporelle". Nous reprenons donc ici les notions traditionnelles de "statif / dynamique", "duratif / ponctuel", "télique / atélique", etc. qui fondent la catégorisation des verbes proposée par Vendler (cf Vendler 1967) ${ }^{13}$. Dans cette catégorisation, les Nev se définissent en premier lieu comme étant de type "dynamique"

\footnotetext{
${ }^{10}$ C'est pourquoi, dorénavant, pour le rattachement de [jusqu'à $\left.\mathrm{SN}\right]$, nous ne parlerons plus de « verbe » ou de « nom » mais de « prédicat verbal » ou de " prédicat nominal ».

${ }^{11}$ Etre et toutes les constructions prédicatives que le verbe peut engendrer : être + Adj, être+ participe passé, être en SN, etc.

12 "éventualité" (traduction littérale de "eventuality" cf. Kamp et Reyle 1993 ou Lascarides et Asher 1993).

${ }^{13}$ C'est la catégorisation que nous adopterons ici, sans en faire une présentation détaillée car dans ce domaine d'étude, c'est sans doute la plus connue et la plus communément adoptée pour les questions d'aspect.
} 
mais se répartissent ensuite entre "ponctuels" et "duratifs", ces derniers se répartissant eux-mêmes entre "atéliques" et " téliques" ${ }^{14}$, ce qui donne lieu à trois catégories distinctes : Activités, Accomplissements et Achèvements.

Ainsi, les Nev que nous nous proposons d'examiner ici sont des noms prédicatifs qui appartiennent à l'une des trois catégories ci-dessous :

Achèvements [-duratif] découverte, démission, libération, expiration, disparition, rupture, etc.

Accomplissements [+duratif] [+télique] réparation, réalisation, transformation, installation, guérison, traduction, évaporation, traversée, etc.

Activités [+duratif] [-télique] déroulement, développement, fonctionnement, croissance, etc

\subsection{La combinaison des propriétés aspectuelles attachées au prédicat et à son complément $S P$ [jusqu'à $S N$ ]}

Sur la base de ces deux types de condition (dynamicité et télicité), on a donc :

- un prédicat (verbal ou nominal) nécessairement duratif, i.e. associé à un déroulement temporel que l'on schématise en général par un axe ou un vecteur orienté - et dont on peut mesurer la durée, soit sur un seul intervalle, pour la durée en continu, soit sur une pluralité d'intervalles, pour l'itération ou la fréquence.

-- un SP [jusqu'à SNev] qui, sur le déroulement temporel qu'exprime le prédicat, fixe une borne - un point d'aboutissement - correspondant, soit à une étape normale dans le développement de la situation dénotée par le prédicat, (22), soit à l'occurrence d'une nouvelle situation qui y met fin, (23) :

(22) (il) me serre la calotte crânienne comme il serrerait une pastèque, jusqu'à l'éclatement.

(23) Jacques leur accordait trente jours de paix, ils pouvaient sommeiller jusqu' à son retour

Le même effet peut être produit avec jusqu'à en construction avec une proposition infinitive ou avec une phrase. Nous pouvons l'illustrer par les exemples (14) et (15) que nous reproduisons ci-dessous :

(14) Ajouter doucement l'eau bouillante en remuant jusqu'à obtenir une pâte épaisse

(15) Vous allez les traquer jusqu'à ce que vous les retrouviez.

Le complément [jusqu'à Nev] peut se lire comme une réponse à une question du type : jusqu'à quand? / jusqu'à quel moment? alors qu'une question s'appli14 Télique ou "intrinsèquement borné" Étant donné les très nombreuses études faites sur
cette catégorisation, il n'est pas nécessaire de donner de plus amples détails ici. 
quant au prédicat dont il dépend, porterait assez naturellement sur la durée : pendant/ pour combien de temps? :

(1) Il dit vouloir poursuivre sa grève de la faim jusqu'à sa libération (=>Jusqu'à quand ?)

Il est à noter que jusqu'à, qui fait du Nev dans le SN une borne de localisation dans le temps, n'impose pas de conditions concernant les propriétés syntaxiques de ce nom; Nev peut être un nom agentif utilisé dans la phrase avec son sujet (24) ou son complément (25) :

(24) Le fournisseur reste propriétaire de la marchandise livrée jusqu'au paiement de l'acheteur

(25) Là il faut mener le décapage jusqu'à l'épuisement complet de la couche archéologique

Mais le Nev peut être également de type ergatif ou inaccusatif ${ }^{15}$ :

(26) Le mode d'emploi dit qu'il faut compter le nombre de gouttes jusqu'au changement de couleur

(27) Maintenez le bouton Marche/Veille enfoncé jusqu'à l'apparition du curseur rouge.

En revanche, des contraintes apparaissent concernant sa valeur aspectuelle : quelle que soit la catégorie à laquelle le Nev se rattache en tant qu'unité lexicale, il doit être capable d'acquérir avec jusqu'à un sens ponctuel, dans la mesure où avec jusqu'à il est utilisé comme une borne - un point d'aboutissement - dans un déroulement temporel.

- Pour les NAchèvement (Nachev), ceci ne fait pas problème puisque précisément, ils sont définis comme des noms ponctuels, ex. découverte, rupture, départ, envoi, acceptation, libération, expiration, offre, refus, etc.

(1) Il dit vouloir poursuivre sa grève de la faim jusqu'à sa libération

(28) Il a dirigé le pays jusqu'à son départ en exil en France en 1986)

Dans ces deux exemples, la situation dynamique propre au prédicat verbal trouve son terme dans l'occurrence de la nouvelle situation représentée par l'événement ou l'action qu'introduit le NAchev.

- Pour un NAccomplissement (NAcc), le sens ponctuel est également possible car s'il est vrai qu'il dénote une action ou un processus impliquant une durée, il

\footnotetext{
${ }^{15}$ Ceci montre qu'avec jusqu'à, la condition de non-agentivité ne joue pas comme elle le fait avec le verbe support, être en voie de, qui marque d'une autre manière la fin d'un déroulement temporel (cf. Borillo 2010).
} 
comporte de manière intrinsèque un aboutissement (un terme). Avec jusqu'à, ce Nacc s'interprète donc le plus souvent comme représentant la phase terminale de l'éventualité, éventuellement suivie d'un état résultatif :

(29) Des soins infirmiers me sont prescrits tous les jours jusqu'à la cicatrisation de cette plaie.

(30) Ce fut une propriété privée puis une exploitation agricole jusqu'à sa transformation par l'Etat en monument national voici un siècle.

Ces noms sont assez souvent modifiés par des adjectifs spécifiant l'idée de complétude, tels que total, complet, parfait, plein, etc. et donc, confirmant par là qu'il s'agit de la phase finale du processus ${ }^{16}$.

(31) Laissez sur le feu jusqu'à la complète absorption de l'eau, la sauce doit être bien consistante et sèche.

(32) Avant l'utilisation du produit, agiter celui-ci jusqu'à sa parfaite homogénéisation

Ces adjectifs accompagnent notamment les NAcc dits $\mathrm{N}$ gradables ${ }^{17}$ (ou scalaires) ex. durcissement, jaunissement, ramollissement, épanouissement, etc. car pour eux, il est parfois difficile de dire si la borne fixée par jusqu'à SN s'applique à l'enclenchement ou à l'aboutissement du processus, cf. (33) :

(33) Il faut arroser régulièrement dés le démarrage de la végétation et ce jusqu'au jaunissement du feuillage vers la fin de l'hiver (=? commence à jaunir)

L'ajout de l'adjectif est souvent là pour préciser :

(34) Les cales doivent rester en place jusqu'au durcissement complet du mastic.

(35) Débrancher l'appareil, retirer la casserole et attendre quelques minutes jusqu'au refroidissement total de la table de cuisson

- Pour les NActivité (Nactiv) qui sont de nature atélique, i.e. qui expriment normalement une action ou un processus ne comportant pas de manière intrinsèque un terme ou un aboutissement, ex. discussion, diffusion, préparation, progression, croissance, utilisation, etc., leur emploi avec jusqu'à leur fait acquérir un sens ponctuel, puisqu'il impose l'idée de borne temporelle. Cependant, les Nactiv peuvent fixer deux moments différents :

- soit le point d'aboutissement fixé par jusqu'à correspond au point de départ, à l'enclenchement de l'action ou du processus. Ces noms prennent alors une valeur inchoative :

${ }^{16}$ Ces adjectifs ne sont pas réservés aux NAcc, ils peuvent modifier également des Nachev «Le passager peut tirer le levier de frein jusqu'à immobilisation totale du véhicule » et même des NActiv (cf. (39) et (40) infra.

${ }^{17}$ L'un des traits qui les caractérisent étant le fait qu'ils peuvent accepter à la fois un complément "en SNdurée" et "pendant SNdurée". 
(36) La laque, récoltée par incision de l'écorce des arbres à laque, est gardée à l'obscurité jusqu'à son emploi.

(37) L'opération doit rester couverte par le secret absolu jusqu'à son déclenchement.

- soit le point d'aboutissement fixé par jusqu'à correspond au moment final de l'action ou du processus ou à son état résultatif :

(38) Les cellules nourricières et folliculaires assurent la croissance et la protection de l'ovocyte jusqu'à sa maturation

Une manière de préciser ceci est précisément d'ajouter au nom un adjectif de la catégorie indiquée plus haut (complet, parfait, total, plein ):

(39) Le liquide obtenu va ensuite être stocké pendant un an jusqu'à sa maturation complète.

(40) Nous nous attachons également à suivre le candidat retenu jusqu'à sa pleine intégration au sein de votre service

Il est à noter cependant que ces adjectifs passent assez mal avec des NActiv qui rendent compte d'une évolution de nature quantitative, ex. augmentation, baisse, diminution, accroissement, etc . Pour eux, la préférence semble aller vers un adjectif comme maximal, le plus fort, etc.

\section{JUSQU'A ET L'ACCESSION AU PLUS HAUT DEGRE D'UNE ECHELLE QUANTITATIVE}

Il existe une autre structure de phrase dans laquelle figure un SP[jusqu'à SN] contenant un nom d'éventualité (Nev) avec la fonction de complément circonstanciel (disjonctif). Mais dans ce cas, jusqu'à ne fait pas du SN régi un point d'aboutissement dans le déroulement d'une action ou d'un processus exprimé par le prédicat, mais un point très élevé, sinon le point le plus élevé, sur une échelle d'évaluation quantitative à laquelle la situation, dans le prédicat, peut être rapportée :

(41) Les bourgeons s'allongent, s'arrondissent, se gonflent jusqu'à l'éclatement

(42) Elle se montrait autoritaire, parfois jusqu'à l'emportement

(43) Agitation et anxiété ont augmenté jusqu'à la perte de contrôle

Dans ce cas, la situation dont rend compte le prédicat, n'est pas nécessairement de nature durative. La dynamicité évoquée par jusqu'à n'est pas associée à un déroulement temporel s'évaluant en termes de durée, mais à une progression ascendante sur une échelle quantitative en termes de degrés. 
Dès lors, [jusqu'à $\mathrm{SN}$ ] est à mettre en correspondance avec la question jusqu'à quel point ? jusqu'à quel degré ? et non pas avec la question jusqu'à quand? jusqu'à quel moment? qui correspond, on l'a vu, à l'emploi «temporel» de jusqu'à que nous venons de présenter au $§ 1$. On peut comparer :

(1) Il dit vouloir poursuivre sa grève de la faim jusqu'à sa libération (=> jusqu'à quand ?)

(43') Agitation et anxiété ont augmenté jusqu'à la perte de contrôle ( $=>$ jusqu'à quel point ?)

Comme pour [jusqu'à SN] que nous dirons, par raccourci, « temporel », il est possible de remplacer ce SP par la proposition [au point de Infinitive] ou par la subordonnée [au point que $\mathrm{P}$ ], pour autant que le $\mathrm{N}$ régi puisse être mis en relation avec un verbe morphologiquement apparenté. Par exemple, (41) pourrait prendre la forme $\left(43^{\prime}\right)$ :

(41') Les bourgeons s'allongent, s'arrondissent, se gonflent au point qu'ils (peuvent) éclater

\section{Examen des propriétés des deux sous-structures mises en relation dans la phrase}

Nous examinerons tout d'abord, quelques propriétés qui peuvent être tenues pour caractéristiques du prédicat auquel le SP [jusqu'à SN] se rattache comme complément, puis dans un deuxième temps, quelques traits concernant le complément lui-même.

\subsection{A propos du prédicat auquel jusqu'à $S N$ se rattache en tant que complément.}

Dans ce deuxième type de phrase, il n'est pas nécessaire que le prédicat soit doté du trait aspectuel [+duratif], que ce trait soit inhérent au sémantisme du Verbe ou du Nom ou qu'il soit dû à des éléments participant à sa construction (temps verbal, auxiliaires, adverbes, etc.). Cette condition, nécessaire, on l'a vu, lorsque le SP [jusqu'à SN] est à interpréter comme un point d'aboutissement temporel (cf. §1. supra), ne tient plus quand le SP marque, pour une situation donnée, un stade ou une propriété figurant un degré élevé, sinon le degré le plus élevé, sur une échelle d'évaluation mesurée en termes d'intensité. Le prédicat auquel se rattache le SP peut être analysé comme une action, un événement ou un processus (Nev), mais également comme un état, et même comme une propriété :

(44) Rien qui ne soit déjà dit sur cet homme d'Etat complexe, admiré jusqu'à l'adoration par ses thuriféraires et haï jusqu'à l'absurde par ses pourfendeurs.

(2) C'est un homme extrême en toutes choses, il aime et il hait jusqu'à la fureur 
(45) Il était. audacieux jusqu'à la témérité, violent jusqu'à la fureur, intrépide jusqu'à la férocité :

Nous pouvons indiquer plusieurs traits marquants qui, d'après les textes ${ }^{18}$ que nous avons consultés, sont assez récurrents et donc peuvent aider à mieux caractériser cette deuxième structure de phrase :

- La présence très courante, dans le prédicat, d'un verbe exprimant la progression, tel que aller (jusqu'à) / grandir (jusqu'à) (cf. Leeman 2005) ou un équivalent causatif, pousser (pousser SN jusqu'à) :

(46) Nous sommes amenés à envisager à votre égard une sanction pouvant aller jusqu'au licenciement

(47) Au cours de la nuit et de la journée, l'enthousiasme a grandi jusqu'au délire.

(48) Il a puisé dans les douloureuses années de sa jeunesse un profond sentiment du devoir qu'il pousse jusqu'à l'abnégation

- La présence, elle aussi assez courante, d'un nom ou d'un adjectif exprimant une propriété ou un état psychologique auquel peut être appliqué une évaluation quantitative :

(49) Chaque fois que les effets de mes calmants baissent, une boule apparait dans ma poitrine, douloureuse jusqu'à la suffocation

(50) Tu es toujours excessive : tantôt gaie jusqu'à l'extravagance, tantôt triste jusqu'à l'abattement (...)

(51) On ne me donne pas de traitement malgré tous les symptômes que j'ai (perte de poids, une très grande fatigue qui va jusqu'à l'épuisement, des vertiges (...)

- L'existence d'un lien sémantique entre le verbe (ou un nom) du prédicat et le nom contenu dans le SP, qui les fait ranger tous les deux dans une gradation croissante sur une échelle d'ordre quantitatif ou intensif. On peut noter ce lien dans les exemples (49) - (51) ci-dessus pour les couples enthousiasme $<$ délire, constance <opiniâtreté, mal de mer $<$ nausée, tristesse $<$ abattement, etc.

\subsection{A propos de jusqu'à et du complément qu'il régit dans le phrase}

Comme cela a été illustré jusqu'ici, le complément apparaît le plus souvent sous une forme nominale, [jusqu'à $\mathrm{SN}$ ]. Mais contrairement à ce qui est exigé dans le cadre du déroulement temporel (cf. §11.b) supra), le nom-tête du SN n'appartient pas nécessairement à la catégorie des Nev. (actions, événements ou processus). Il est assez fréquent qu'il renvoie à la catégorie "Etat", et plus particulièrement aux sous-catégories que l'on peut caractériser comme "état physique », « état psychologique », « sentiment », etc. :

(52) Je suis submergé de compliments, jusqu'à l'écœurement

(53) Elle se savait attendue par un peuple aimant et enthousiaste jusqu'au délire

\footnotetext{
${ }^{18}$ Voir la note 8 supra donnant l'origine de ces textes.
} 
(54) Ici, j'ai goûté la solitude souvent jusqu'à l'ivresse, rarement jusqu'à la satiété

Il peut aussi relever d'une catégorie abstraite et renvoyer à des propriétés d'ordre cognitif, moral ou conceptuel :

(55) Il est poussé à réaliser des esquisses où sa peinture est simplifiée jusqu'à l'abstraction

(56) La petite fille s'exerce sans relâche, répétant chaque geste jusqu'à la perfection

Indépendamment de sa catégorie, concrète ou abstraite, le nom peut participer à une expression figée, dans laquelle s'exprime de manière métaphorique l'idée d'intensité portée à un degré extrême, ex. jusqu'au sang, jusqu'à la lie, jusqu'à l'os, jusqu'à la corde. jusqu'à la folie, jusqu'à satiété, jusqu'à plus soif, etc. :

(57) L'avide curiosité qui pousse le lecteur à se gorger jusqu'à satiété (...)

(58) Je me mordais jusqu'au sang la lèvre inferieure pour ne pas rire

Enfin, comme on l'a déjà dit au tout début $(\S 1)$, le SN peut laisser la place à une proposition infinitive [jusqu'à Infinitive] dont le sujet implicite est co-référent avec celui de la phrase dont elle dépend :

(59) Parfois le ton montait brusquement, jusqu'à atteindre le cri

Dans ce deuxième type de structure, l'emploi de l'infinitif est beaucoup plus courant que lorsque jusqu'à s'inscrit dans le cadre d'un déroulement temporel (cf. les exemples (14) et (16) du §1). Et en tout cas, il n'apparaît pas comme relevant d'un usage marqué :

(60) Il serrait les poings jusqu'à faire jaillir un peu de sang sous ses ongles

On voit en particulier que cette construction est très communément associée à des verbes traduisant la dynamicité, comme aller (jusqu'à), pousser (jusqu'à), s'avancer (jusqu'à), grandir (jusqu'à), etc. que nous avons mentionnés plus haut (cf. (46) à (48)) :

(61) J'ai poussé l'audace jusqu'à poser des questions qui auraient pu paraître inconvenantes

(62) Je ne pourrais pas m'avancer jusqu'à dire qu'elle retrouvera un jour toute sa raison
aiso pourrais pas m'avancer jusqu'à dire qu'elle ret

(63) Il a poussé la complaisance jusqu'à ouvrir la porte

Il est certain qu'une étude plus approfondie serait à faire pour ce deuxième type de construction, dont nous venons juste de dessiner quelques grands traits. 


\section{EN GUISE DE CONCLUSION :}

Si l'on considère les deux types de construction qui ont été retenus pour illustrer deux emplois différents de jusqu'à - l'un où il marque un point d'aboutissement dans un déroulement temporel, l'autre où il pointe vers un degré très élevé, sinon maximal, sur une échelle d'évaluation quantitative - on constate que leurs caractérisations sont relativement différentes et que les effets de sens qui en découlent sont assez éloignés.

Cependant, il peut arriver que pour une même phrase, les deux lectures de jus-

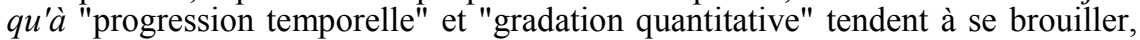
au point qu'on peut hésiter parfois entre l'une ou l'autre interprétation ou qu'on accepte plus ou moins l'amalgame. Ceci est particulièrement le cas lorsque un prédicat possède à la fois le trait +duratif et le trait +dynamique, ex (64) :

(64) Le développement de l'humanité ralentit jusqu'à la stagnation

(65) Les liquidateurs, véritables kamikazes de l'atome, s'étaient dépensés jusqu'à l'épuisement

Dans ces deux phrases, on peut interpréter le $\mathrm{SP}$ [jusqu'à $\mathrm{SN}$ ] de deux manières un peu différentes : soit comme « point d'aboutissement dans un déroulement temporel » et dans ce cas (64) et (65) peuvent respectivement se lire comme :

(64') Le développement (...) ralentit jusqu'au moment où il commence à stagner

(65) Les liquidateurs (...) s'étaient dépensés jusqu'au moment où qu'ils avaient atteint l'épuisement

soit comme « point maximal dans une gradation quantitative » et dans ce cas, on peut lire ces deux phrases comme :

$(64$ '") (...) connaît un ralentissement qui s'accentue jusqu'à la stagnation

$\left(65^{\prime \prime}\right)(\ldots)$ avaient fourni une dépense de forces qui déclinent jusqu'à l'épuisement

Cette double lecture (dont les deux sens finalement ne s'excluent pas) est due à une conjonction de propriétés, à la fois d'ordre syntaxique et sémantique, qui caractérisent à la fois le prédicat et le $\mathrm{SP}$ [jusqu'à $\mathrm{SN}$ ] . Ces propriétés présentes dans les deux phrases ci-dessus, peuvent être résumées ainsi :

- dans [jusqu'à SN]. le nom est un Nev,

- le prédicat a le trait [+duratif] et est susceptible d'avoir un complément temporel répondant à "jusqu'à quand ? Mais en même temps, il peut recevoir un complément de gradation quantitative répondant à "jusqu'à quel point?

- une co-référence existe entre le sujet du prédicat et le sujet du Nev, 
- un lien sémantique peut être établi entre un élément du prédicat et le Nev dans le SP, les plaçant dans un ordre croissant sur une échelle de mesure quantitative ralentir $<$ stagnation, se dépenser $<$ épuisement.

On peut comprendre que les deux exemples donnés en (64) et (65) représentent des cas d'exception, car il est très rare que les propriétés détaillées ci-dessus se trouvent toutes réunies dans une même phrase. Il suffit qu'une ou deux de ces propriétés ne soient pas présentes, et le sens à donner à la phrase devient tout de suite plus clair. Si l'on examine, par exemple, une phrase comme (66) cidessous, où le prédicat dont dépend jusqu'à ne s'interprète pas avec le trait [+duratif] mais apparaît comme traduisant une propriété mentale, le sens qui est donné à [jusqu'à $\mathrm{SN}$ ], concerne moins la progression dans un déroulement temporel que l'accession à un haut degré sur le plan quantitatif :

(66) On note une perte de la cohésion, de l'efficacité de la pensée qui apparaît embrouillée, désordonnée, souvent engourdie jusqu'à la stagnation

Si l'on prend un peu de recul, et si l'on se dégage de l'ensemble des propriétés que jusqu'à requiert et met en œuvre pour fonctionner comme complément dans chacun des deux types de structure de phrase, on voit qu'il lui reste en propre un rôle de marqueur aspectuel de type télique exprimant l'idée " d'aboutissement », «d'accession » à un terme fonctionnant comme limite, que celle-ci soit à inscrire dans une dynamique temporelle ou dans un développement quantitatif.

\section{REFERENCES BIBLIOGRAPHIQUES}

Adler, S. \& M. Asnes, 2004, Les compléments de degré en « jusqu'à », Travaux de linguistique, 49, p.103-159.

Anscombre, J.-C, 1973, Même le roi de France est sage, Communications 20, p. 40-82.

Borillo, A., 2005, Quelques formes lexicales de l'aspect adnominal en français Cahiers de grammaire 30, p. 21-38.

—, 2010, L'ambiguïté des Noms d'Eventualités (Nev) à la lumière de certaines constructions aspectuelles, Proceedings of the 29th International Conference on Lexis and Grammar, Belgrade, p.27-38

Ducrot, O., 1980, Les échelles argumentatives, Paris, Les Éditions de Minuit.

Fortis, J-M., 2006, Les fonctions de «jusqu'à, » Modèles linguistiques, 54, p.137-154.

Kamp, H. \& U.Reyle, 1993, From discourse to logic, Dordrecht / Boston, Kluwer.

Leeman, D., 2005, La préposition « jusque », in P. Dendale (éd) Le mouvement dans la langue et la metalangue, Recherches linguistiques, 27, p. 103-119.

- 2005, Un nouvel auxiliaire " aller jusqu'à », dans H. Bat-Zeev Schyldkrot et N. Le Querler (éds) Les périphrases verbales, Amsterdam, John Benjamins, p. $423-434$.

Vendler, Z., 1967, Linguistics in Philosophy, Ithaca, Cornell University Press. 